\title{
Stoichiometric Low Loss Tellurium Oxide Thin Films for Photonic Applications
}

\author{
Khu T. Vu ${ }^{1}$, Steve J. Madden ${ }^{1}$, Barry Luther-Davies ${ }^{1}$, and Douglas Bulla ${ }^{1}$ \\ ${ }^{1}$ Laser Physics Centre, Research School of Physical Sciences and Engineering, The Australian National University, \\ Canberra, ACT 0200, Australia \\ Phone: +612 6125 4079, Fax: +6126125 0029, Email: khu.vu@anu.edu.au
}

\begin{abstract}
Stoichiometric low loss Tellurium Oxide, $\mathrm{TeO}_{2}$, films have been produced by reactive $\mathrm{RF}$ sputtering. $\mathrm{TeO}_{2}$ films with propagation loss below $0.1 \mathrm{~dB} / \mathrm{cm}$ at $1550 \mathrm{~nm}$ have been achieved in as deposited films.
\end{abstract}

\section{Introduction}

Tellurite glasses are glasses with Tellurium Oxide $\left(\mathrm{TeO}_{2}\right)$ as the main component. Tellurite glasses have some additional attractive properties for nonlinear optics compared with the widely investigated Silica and Chalcolgenide glasses. These include high accoustooptic figure of merit [1]; high nonlinearity (up to $100 \mathrm{x}$ silica); a large Raman shift (up to 2.5x that of silica); and good chemical stability [2]. These glasses also have very wide transmission window from the UV at $\sim 300 \mathrm{~nm}$ up to mid-infrared at $\sim 5000 \mathrm{~nm}$. Thin films of tellurites have been produced by chemical and sol-gel processes [3]; thermal evaporation [4]; reactive RF sputtering using pure Tellurium [5]; and pulsed laser deposition [6].

Obtaining stoichiometric, low loss Tellurite thin films is crucial for any future application in integrated nonlinear photonics. In this paper, we report on the use of reactive RF sputtering that results in films with the required properties. We also report the effects of annealing on the films.

\section{Experimental}

$\mathrm{TeO}_{\mathrm{x}}$ thin films were produced by reactive RF sputtering from a pure Te target in an $\mathrm{O}_{2} / \mathrm{Ar}$ gas mixture. The sputtering system was an AJA International A300. The Te target was a powder pressed plate $(99.95 \%)$. The rotating substrates placed at a distance of $\sim 20 \mathrm{~cm}$ from the target and kept at room temperature. The sputtering parameters that were investigated were chamber pressure (2.0mTorr to $20 \mathrm{mT}$ Torr), RF power (120W to $360 \mathrm{~W}$ ), and percentage of Oxygen flow (20\% to $80 \%)$ - the total flow of $\mathrm{O}_{2}$ and Ar was kept at $15 \mathrm{sccm}$.

The films so obtained (up to $2 \mu \mathrm{m}$ thick) were characterised using EDXA (Jeol 6400 SEM) for composition; by a dual angle spectroscopic reflectometry for thickness and refractive index (SCI Filmtek 4000); and by prism coupling for optical losses via image processing of the light streaks observed using a Sensors Unlimited SU-320 IR camera.

A series of $\mathrm{TeO}_{\mathrm{x}}$ films at various $\mathrm{O}_{2}$ flow around the optimum conditions was fabricated. This series of films were then characterised fully to determine the relation between the composition, refractive index and loss.
Some of the samples were then annealed in an oven filled with air at 200C for durations up to 33 hours. The refractive indices of the samples were monitored during annealing and the compositions were measured at the end to compare with the initial condition. X-ray diffraction was also used to detect any crystallisation during the annealing process.

\section{Results and Discussion}

As a wide range of sputtering parameters were used during film deposition. The as-deposited amorphous $\mathrm{TeO}_{\mathrm{x}}$ films were classified into three types: Tellurium rich $(x<2)$; close to stoichiometric $(x \sim 2)$; and Oxygen rich $(x>2)$. Tellurium rich films have excessive levels of metallic atoms and are therefore highly absorbing. These films have an effective band edge high up in the red or infrared and refractive indices at $1550 \mathrm{~nm}$ greater than 2.1. Tellurium rich films are produced when there is an Oxygen deficiency in the chamber, or a high flux of Tellurium from the target. This condition occurs at high RF power, low Oxygen flow or low chamber pressure. Oxygen rich films on the other hand are highly transmissive down to the violet or UV, have low absorption in the infrared as well as lower density and refractive index at $1550 \mathrm{~nm}$ below 2.1 . These films were produced when the sputtering power was low; the Oxygen flow or chamber pressure high. Stoichiometric films were obtained when the balance of all three parameters was correct which corresponded to $5 \mathrm{mT}$ Torr pressure; $150 \mathrm{~W}$ RF power; and 45\% Oxygen flow. These films were found not only to have the correct stoichiometry but also low loss.

The propagation losses at $1550 \mathrm{~nm}$ were determined from observation of the light streaks (figure 1) for a set of as-deposited $\mathrm{TeO}_{\mathrm{x}}$ thin films on silica substrates with film thickness of around 1.5um and composition $\mathrm{O} / \mathrm{Te}$ ratios of 1.6 to 2.4. The results are shown in Figure 1. As is evident, the minimum loss was $<0.1 \mathrm{~dB} / \mathrm{cm}$ at $1550 \mathrm{~nm}$, which is the first time such low losses have been observed for an as-deposited film. As argued above, an excessive level of Te in the films, $x<2$, is expected to produce high losses and this is indeed observed. However, the loss curve dips to a minimum right at the stoichiometric point before gradually increasing as the Oxygen content increases. The losses of Oxygen rich films would be expected to be lower when they are annealed to remove stress [7].

In general thin films deposited onto cold substrates are expected to be far from a minimum entropy state 
immediately after deposition. Hence thermal annealing is often used to "relax" the films. Furthermore, there are reports of annealing Te rich films in oxygen to oxidise the excess Tellurium thereby reducing loss and risk of crystallization [8]. With this in mind we annealed the films in Oxygen at temperatures up to $300 \mathrm{C}$.

In order to observe the behaviours of films with excess Oxygen during annealing, a set of samples that had Oxygen to Tellurium ratios of 2.04, 2.29, 2.5 and 3.24 were annealed in an air-filled oven for durations of more than 33 hours. The refractive indices were measured at different times and are shown on Figure 2.

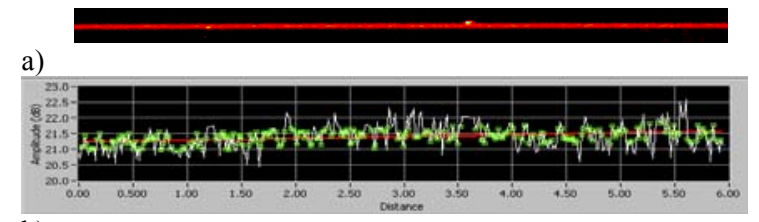

b)

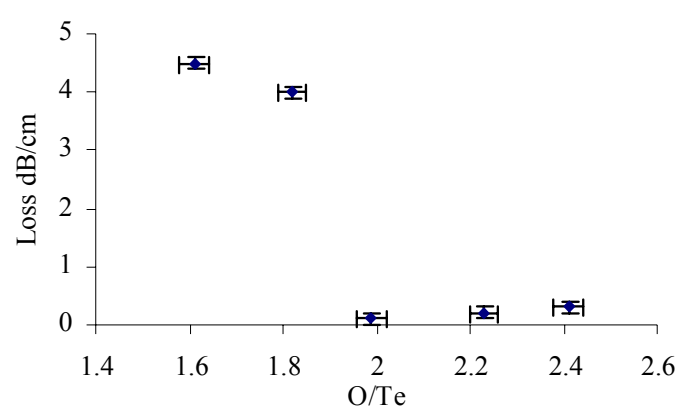

c)

Figure 1: a) light streak image acquired by the camera, in the $\mathrm{O} / \mathrm{Te}=2.0$ film for a length of $6 \mathrm{~cm}$, light propagates from right to left; b) data analysis with fitting to obtain a slope of less than $0.1 \mathrm{~dB} / \mathrm{cm}$; c) propagation loss at various $\mathrm{O} / \mathrm{Te}$ ratios.

There are significant trends evident in the plots. For an $\mathrm{O} / \mathrm{T}$ ratio of 2.5 there was essentially no change in refractive index upon annealing, which is an interesting result not previously reported for $\mathrm{TeO}_{2}$ thin films. Away from this composition annealing occurred but was complete within abut 500 minutes. Increases in refractive index upon annealing are generally explained by the rearrangement of atoms to a more relaxed, lower energy state with higher density. However, in this particular case, there were competing processes that decreased the refractive indices in correlation with the observed increase in the thickness of the films.

EDXA measurements at the end of the annealing study showed unchanged overall composition. Furthermore, X-ray diffraction measurements suggested that the films maintained their amorphous phase. Therefore the changes observed were not brought about by either oxidation or crystallisation.

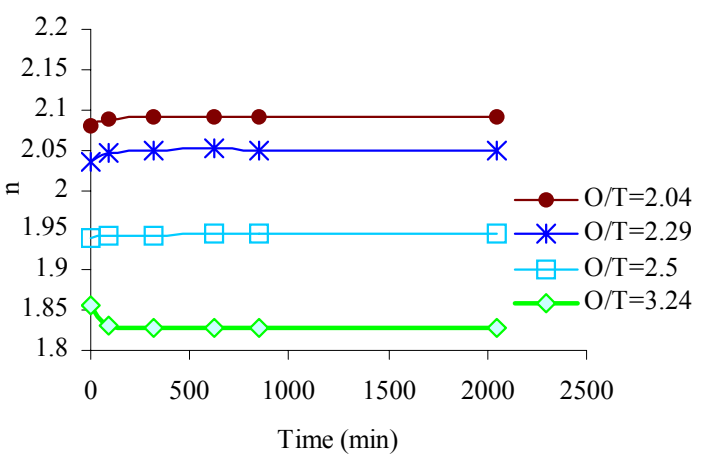

Figure 2: Effect of annealing at $200 \mathrm{C}$ on the refractive indices of samples at different compositions.

\section{Conclusions}

We have studied reactive RF sputtering of Tellurium in $\mathrm{O}_{2}$ and $\mathrm{Ar}$ gases showing that it is possible to obtain stoichiometric, low loss Tellurium Oxide, $\mathrm{TeO}_{2}$, films. Propagation losses $\leq 0.1 \mathrm{~dB} / \mathrm{cm}$ at $1550 \mathrm{~nm}$ were achieved in as-deposited films for the first time. Annealing studies also reveal the possibility of making films that are totally thermally stable, clearly an interesting development for future applications.

\section{References}

1. N. Uchida and Y. Ohmachi, J. Appl. Phys. 40 (12) (1969) p.3736

2. R. A. H. El-Mallawany, Tellurite Glasses Handbook: physical properties and data, (2002) CRC Press

3. S. N. B. Hodgson and L. Weng, J. Mater. Sci. - Mater. Electron. 17(9) (2006). p.723

4. M. F. Al-Kuhaili et al, J. Phys. D: Appl. Phys. 35(9) (2002) p.910

5. N. Dewan et al, J. Appl. Phys. 101(8) (2007) p.084910

6. A. P. Caricato et al, Mater. Sci. Eng. B 105 (2003) p.65

7. R. Nayak et al, Thin Solid Films 445(1) (2003) p.118

8. A. Singh and R. A. Lessard, Solid State Commun. 51(11) (1984) 845-848. 